\title{
Comments on clinical outcomes of sigmoid colon volvulus: identification of the factors associated with successful endoscopic detorsion
}

\author{
Sabri Selcuk Atamanalp \\ Department of General Surgery, Ataturk University Faculty of Medicine, Erzurum, Turkey
}

I read with interest the article by Iida et al. ${ }^{1}$ who reported the factors affecting the success of endoscopic detorsion in sigmoid volvulus (SV). The subject raised by the authors is extremely interesting. Among a large number of evaluated criteria, the absence of abdominal tenderness, the use of laxatives, and the history of open abdominal surgery have been reported as predictive factors for successful endoscopic detorsion.

I practice in eastern Turkey, which is an endemic SV area. ${ }^{2}$ My clinic has 51 years of history and 1,000 cases of endoscopic and/or surgical experience with SV. This is the largest single-center SV series in the world according to the literature in major research databases, including Web of Science ${ }^{3}$ and PubMed. ${ }^{4}$ In our series, the success rate of endoscopic detorsion is $77.5 \%$, and increases to $82.4 \%$ when gangrenous cases are excluded. In my experience, in addition to the elements presented by Iida et al., ${ }^{1}$ there are 3 important factors causing unsuccessful endoscopic detorsion: a prolonged symptomatic period, the presence of over-rotation, and the co-occurrence of ileosigmoid knotting (ISK). It is well known that these factors also lead to the development of bowel gangrene in $\mathrm{SV}^{5}$

Although ISK, which is a complex volvulus of ileum and sigmoid colon, is a component of SV, it is generally considered a different entity, and is not evaluated in SV series. ISK is principally treated with emergency surgery, and when

Received August 25, 2017. Revised August 28, 2017.

Accepted August 28, 2017

Correspondence to Sabri Selcuk Atamanalp, Department of General

Surgery, Ataturk University Faculty of Medicine, 25040 Erzurum, Turkey.

Tel: +90-442-3447560, Fax: +90-442-3446528, E-mail: ssa@atauni.edu.tr

Financial support: None. Conflict of interest: None. diagnosed accurately using CT or MRI, endoscopic detorsion is not tried. ${ }^{6}$ Consequently, there are no ISK cases in the series presented by Iida et al. ${ }^{1}$ Nevertheless, I really wonder about the role of the prolonged symptom period and overrotation in the failure of endoscopic detorsion in their series. In my opinion, the presence of a long period between the onset of symptoms and endoscopy may cause bowel and mesentery edema, which theoretically may hinder detorsion. I think that the records of symptom duration should be reevaluated in the retrospective series presented by Iida et al. ${ }^{1}$ Finally, in my opinion, the presence of over-rotation, which is described as rotation greater than $360^{\circ}$, may block detorsion mechanically, and may cause an endoscopic washout. As discussed by Iida et al., ${ }^{1}$ abdominal CT has been used in the diagnosis of these patients. In my experience, although the amount of rotation is best determined via surgery, it can also be estimated via CT. I hope these 2 subjects will be reevaluated by the authors. If not possible, I would at least appreciate the authors' thoughts on my comments.

I congratulate the authors on their interesting study and look forward to their reply.

\section{REFERENCES}

1. Iida T, Nakagaki S, Satoh S, Shimizu H, Kaneto H, Nakase H. Clinical outcomes of sigmoid colon volvulus: identification of the factors associated with successful endoscopic detorsion. Intest Res 2017;15:215-220.

2. Atamanalp SS. Sigmoid volvulus: diagnosis in 938 patients over 45.5 years. Tech Coloproctol 2013;17:419-424.

- Copyright 2017. Korean Association for the Study of Intestinal Diseases. All rights reserved.

This is an Open Access article distributed under the terms of the Creative Commons Attribution Non-Commercial License (http://creativecommons.org/licenses/by-nc/4.0)

which permits unrestricted non-commercial use, distribution, and reproduction in any medium, provided the original work is properly cited. 
3. Sigmoid volvulus. Web of Science Web site. http://apps. webofknowledge.com/WOS_GeneralSearch_input. do?product=WOS\&search_mode=GeneralSearch. Accessed August 01, 2017.

4. Sigmoid volvulus. PubMed Web site. https://www.ncbi.nlm.nih. gov/pubmed/. Accessed August 01, 2017.
5. Atamanalp SS, Kisaoglu A, Ozogul B. Factors affecting bowel gangrene development in patients with sigmoid volvulus. Ann Saudi Med 2013;33:144-148.

6. Atamanalp SS. Treatment for ileosigmoid knotting: a singlecenter experience of 74 patients. Tech Coloproctol 2014;18:233237. 